\title{
Injuries of Non-Lethal Child Physical Abuse to the Crania and Orofacial Regions: A Scientific Review
}

\author{
Banheiro $\mathbf{R}^{1}$, Pereira Escobar $\mathbf{C}^{2}$ and Cristiana Palmela Pereira $\mathbf{C}^{3,4^{*}}$ \\ ${ }^{1}$ Private Practitioner, Master Thesis, Dental Faculty from University of Lisbon, Lisbon, Portugal \\ ${ }^{2}$ Trainee Lawyer of GAP Lawyers, Master Student at the ISCTE-IUL, Lisbon, Portugal
}

${ }^{3}$ Auxiliary Professor, Dental Faculty, University of Lisbon, Scientific Consultant of Forensic Dentistry South Branch from Portuguese National Institute of Legal Medicine and Forensic Sciences, Lisbon, Portugal

${ }^{4}$ Integrated Research from the Centre of Statistics and Applications of University of Lisbon (CEAUL), Portugal

*Corresponding author: Pereira CP, Auxiliary Professor, Dental Faculty, Scientific Consultant of Forensic Dentistry, University of Lisbon, Portugal, Tel: 00351914796217; E-mail: cristiana.pereira@fmd.ul.pt

Rec date: Dec 19, 2014 Acc date: April 03, 2015 Pub date: April 07, 2015

Copyright: @ 2015 Banheiro R, et al. This is an open-access article distributed under the terms of the Creative Commons Attribution License, which permits unrestricted use, distribution, and reproduction in any medium, provided the original author and source are credited.

\begin{abstract}
The literature states that maltreatment in childhood and youth make up a problem on a global scale that exceeds ethnicities, religions, cultures, social and economic classes. It is also said that more than half of the injuries from maltreatment occur in the head and face. Assuming the particular relevance of orofacial structures, the dentist must know how to observe and recognize the indicators and properly diagnose the injury by maltreatment. This scientific review aimed to understand what types of intraoral lesions, signs or external lesions of the head or neck can be associated with child maltreatment. One primary database was searched so that systematic review articles and meta-analysis, case reports or case series of intraoral lesions, signs or external lesions of the head or neck by child maltreatment could be acquired. The references in the works acquired by electronic search were manually researched and the authors of all possibly relevant papers were contacted. In all searches inclusion and exclusion criteria were applied. Of the twenty two papers included two were systematic reviews and twenty were case reports or case series. The twenty articles of case reports or case series exposed information from thirty-five clinical cases included. Despite the limitations of scientific evidence it can be concluded that oral cavity, head and neck regions are home to multiple and diverse injuries by maltreatment of children and youngsters.
\end{abstract}

Keywords: Scientific review; Physical abuse; Children; Injuries; Crania; Orofacial

\section{Introduction}

According to the American Child Abuse Prevention and Treatment Act, child abuse and neglect is a recent act or failure to act on the part of a parent or caretaker, which results in death, serious physical or emotional harm, sexual abuse or exploitation, or an act or failure to act which presents an imminent risk of serious harm prevailing four main forms of child abuse: physical abuse, sexual abuse, emotional abuse and neglect [1-3]

First described by John Caffey the shaken baby syndrome is a devastating form of abuse with clinical characteristics appearing in triad and including retinal, subdural and/or subarachnoid haemorrhage in children with non or few external signs of trauma [4,5]. Another particular form of abuse is Munchausen syndrome by proxy in which the child's caregivers produce the signs and symptoms of a disease, not having this syndrome a typical presentation [6]. Suspicions may arise when parents don't correctly interpret or exaggerate normal behaviours, ranging cases between apparent fabrications to the provocation of the symptoms [6].

In both short and long term maltreatment can affect adversely children's health and development $[7,8]$. Injuries by maltreatment, particularly traumatic brain injury, can result in significant long-term disability including seizures, spasticity, blindness, paralysis and mental retardation [1]. However, minor injuries such as those inflicted on physical discipline not requiring medical care can be hidden [1]. Maltreatment in childhood comprises a problem of global scale that exceeds ethnicities, religions, cultures, social and economic classes [2]. Any situation that conditions an increased likelihood of maltreatment of a child is considered a risk factor for maltreatment [2]. Considered to be indicators of abuse are the signs and symptoms or changes in behaviour (psychological indicators) that when present rise suspect over the existence of an abusive situation [2].

As with any other type of medical work, the exam must be thorough and documented [9]. The purpose of the examination should be clear: to make the temporary or definitive diagnosis of maltreatment [9]. Not intended to decide what happens to the child-it is function of the social services; or identify the perpetrator-it is function of the police [9]. The detection of child maltreatment depends on clinicians' ability to recognize and diagnose suspicious lesions, conduct a complete physical examination, with judicious use of diagnostic exams, and consider whether the explanation given by caregivers is supported by the characteristics of the injury or injuries and developmental capabilities of the child $[10,11]$. It should also be taken into account how often the child is observed by trauma [12].

Most orofacial injuries reported in literature consist of dental fractures, frenulum lacerations inflicted during forced feeding, missing teeth for no apparent reason, abnormal appearance and mobility of the tongue, maxillary and mandibular fractures [13,14]. Other manifestations include contusions, lacerations, abrasions, burns, oral trauma, traumatic alopecia and bite marks $[15,16]$. 
Injuries in relatively protected sites raise suspicion of abuse, especially if such lesions are extensive and vary in age. In practice, dating injuries is inaccurate [15]. The absolute age of lesion is virtually impossible to estimate, abrasion, and sensitivity or edema suggest a recent injury [7]. It can be said that bruises with less than 24 hours show no yellow tint, but red bruises may persist for a week [15]. Another important factor is that lesion may reflect the shape of the object used to inflict them [15]. Lesions with defined patterns are a strong indicator of abuse, as are multiple injuries, clustered, with unusual locations, distant from bony prominences and in different stages of healing $[8,15]$. There are, however, conditions that predispose to bleeding, such as disease Von Willbrand among other forms of anaemia and should be considered in the differential diagnosis of lesions $[18,19]$.

\section{Objectives}

The aim of this scientific review was to answer the question: In children and youngsters of both sexes victims of non-fatal maltreatment what types of intraoral lesions, signs or external lesions of the craniofacial can be associated with the diagnosis of maltreatment?

\section{Materials and Methods}

MeSH terms for "child", "maltreatment" and "mistreatment" were probed, these being the terms of the expression "child physical abuse".

\section{Electronic search for prior meta-analyses and systematic reviews}

A research for meta-analyses or systematic reviews prior to this study was conducted on PubMed primary database (http:// www.ncbi.nlm.nih.gov/pubmed). Two combinations of terms, "child physical abuse" and "battered child syndrome", were used, having been applied filters for "systematic review", "meta-analysis", "full text", "10 years", "human", "English", "French", "Portuguese", "Spanish" and "child: birth to 18 years". Titles and abstracts were revised and classified at this stage as "irrelevant" or "possibly relevant". Papers that were not, as intended, systematic review or meta-analysis, that didn't describe intraoral lesions, signs or external lesions of the head or neck by maltreatment, works not published in the languages mentioned above, related to adults, that dealt exclusively with deadly abuse and dual publications were considered irrelevant and excluded. Of the 116 references yielded only one was considered possibly relevant. The systematic review obtained was assessed using the Critical Appraisal Skill Programme (available in http://www.casp-uk.net/) and having rated higher than $50 \%$ was considered "relevant" and included.

\section{Electronic search for case reports and case series}

The same combinations of terms were used on PubMed database to obtain case reports and case series having filters for "case reports", "full text", "from 01.01.1984 to 07.15.2014", "human", "English", "French", "Portuguese" "Spanish" and "child: birth to 18 years" been applied. The titles and abstracts of the articles were revised and classified as "irrelevant" or "possibly relevant". Papers that weren't, as intended, case reports or case series, that didn't describe intraoral lesions, signs or external lesions of the head or neck by maltreatment, works not published in the languages above mentioned, related to adults, that dealt exclusively with fatal maltreatment or whose evidence of maltreatment was considered weak and dual publications were classified as irrelevant and excluded from the review. Of the 373 references yielded 19 were considered possibly relevant. Case reports and case series publications considered possibly relevant were analysed having been classified as "relevant" and included the case reports that describe intraoral lesions, signs or external lesions of the head or neck by child maltreatment, that were related to children or youngsters (under 18 years old), cases that didn't describe fatal maltreatment and whose evidence of maltreatment was considered strong. Inclusion and exclusion criteria applied to case reports analysis are described in Table 1. In Table 2 it is described maltreatment evidence classification.

\begin{tabular}{|l|l|}
\hline Criteria & Description \\
\hline Inclusion & Intraoral lesions, signs or external lesions of the head or neck by maltreatment are described. \\
\hline Exclusion & Related to adults (above 18 years old). \\
\hline Exclusion & Lethal child maltreatment described. \\
\hline Exclusion & Weak evidence of maltreatment. \\
\hline
\end{tabular}

Table 1: Inclusion and exclusion criteria applied to case reports.

\begin{tabular}{|l|l|}
\hline Classification & Description \\
\hline Strong & $\begin{array}{l}\text { Maltreatment confirmed in court; suspicion that resulted in } \\
\text { activation and action of the social services for minors } \\
\text { protection; agreement over the diagnosis among peers; } \\
\text { perpetrator's confession or testimonies. }\end{array}$ \\
\hline Weak & Suspicious or temporary diagnose ("most likely"). \\
\hline
\end{tabular}

Table 2: Classification and description of the evidence of maltreatment.

\section{Manual search and authors contact}

All references cited on works considered "relevant" and "possibly relevant" were scouted and those with apparent relevancy were searched in PubMed database accessing the abstracts. These papers were analysed as described before for electronic search. Nine case reports or case series were considered "possibly relevant" and one systematic review was considered "relevant". The authors of "relevant" and "possibly relevant" papers were contacted.

For statistical analysis it was used SPSS software version 22 of IBM ${ }^{\bullet}$. 


\section{Results}

After searching in electronic database 489 titles and abstracts underwent analysis, having twenty been considered "relevant" or "possibly relevant". In manual search 271 titles and abstracts were analysed, having ten been considered "relevant" or "possibly relevant". $55 \%$ of the author responded to first contact, not having been added new papers. From thirty papers full text versions of twenty eight were obtained. Neither injuries reported by negligence or sexual abuse nor racial characteristics and socio-economic context of the abused children were analysed or described. Two systematic reviews were included $[20,21]$. Seventy one clinical cases were reported in twenty six papers; thirty five met the inclusion criteria having thirty six been excluded [22-47]. The exclusion of all clinical cases described in one paper resulted in the exclusion of such paper, having six been excluded $[25,30,34,37,38,44]$.

\section{Systematic reviews analysis}

There are differences between unintentional injuries and those observed in child abuse [20]. Lesions in infants without independent mobility are very uncommon (less than $1 \%$ ), while the majority of children in preschool and school age presents accidental injuries [20] Accidental injuries are typically small and frequently on bony prominences, with prevalence, number and location of the injuries directly related to motor development [20]. Any part of the body is vulnerable to lesions by maltreatment, with lesions being located far from bony prominences [20]. Lesions by maltreatment are often larger, multiple and observed in clusters [20]. Frequently associated with lesions that may be older and may contain the pattern of the weapon used [20]. Intraoral lesions occur in a significant number of abused children, being widely distributed (lips, mucosa, tongue and palate) [21]. Frenulum laceration is mainly described in children of preschool age target of grievous abuse [21]. As the literature mentions accidental frenulum laceration a laceration isolated cannot be described as pathognomonic of child maltreatment [21].

\section{Case reports analysis}

The age of the abused children varied between nine days and thirteen years with average age of 27, 19 months and standard deviation of 391 months. Fourteen children were female (40\%) and twenty one were males $(60 \%)$, with no relevant statistical difference (T-test, $\mathrm{p}=0.084$ ).

The lesions were distributed through face, head, neck and intraoral region with significant statistical differences (non-parametric test: Q Cochran Test for related sample, with $\mathrm{p}=0,000)$ : $85.7 \%$ of the cases reported facial lesions, $25.7 \%$ reported intraoral lesions, $17.1 \%$ reported head lesions and $8.6 \%$ reported neck lesions. Statistical differences of lesions distribution within oral cavity were irrelevant ( $p=0.881$; non-parametric test: $\mathrm{Q}$ Cochran Test for related sample), having been reported lip contusions, gingiva and mucoalveolar lacerations, teeth avulsions and intrusions, hard palate abrasion, frenulum lacerations, pharynx lacerations and perforations, soft palate contusions and lacerations, oral bleeding, pharynx excoriations, mouth floor lacerations and traumatic absence of teeth.

Several types of lesions were described in the craniofacial and intraoral regions: traumatic absence of teeth, teeth avulsions and intrusions, petechiae, ecchymosis, contusions, lacerations, punctures or abrasions, bruising, swelling, scars, burns, subconjunctival haemorrhage and chemosis hyphema, oral bleeding, nosebleeds and bloody otorrhea. Concerning dental trauma, no statistical differences ( $\mathrm{p}=000$; non-parametric test: $\mathrm{Q}$ Cochran Test for related sample) between avulsions, intrusions and traumatic absence of teeth were noted.

No statistical differences between petechiae and ecchymosis were observed in the literature ( $\mathrm{p}=0.625$; non-parametric test: McNemar Test for related sample). Contusions were reported in $40 \%$ of the 35 clinical cases, being the most described type of lesion in the reviewed literature. Hematomas, however, were referred in $8.6 \%$ of case reports.

Between lacerations, perforations and excoriations significant statistical differences were noted $(\mathrm{p}=0.03$, non-parametric test related sample Cochran Test), having lacerations been referred in $31 \%$ of case reports. Edema was described, as were scares and buns, not having been seen significant statistical differences between the lasts ( $p>0.05$, non-parametric test). Between the several kinds of bleedings (subconjunctival, oral, nosebleeds and bloody otorrhea) and ophthalmological signs (chemosis and hyphema) there weren't significant statistical differences $(\mathrm{p}=0.904$, non-parametric test $\mathrm{Q}$ Cochran for related samples) being subconjunctival haemorrhage and bloody otorrhea the most referred (14\%). Bloody otorrhea was referred as sign and symptom of Munchausen syndrome by proxy in four case reports.

\section{Discussion}

The aim of this review was to understand which intraoral lesions, signs or external lesions of the craniofacial may be associated with non-lethal maltreatment of children and youngsters. In fulfilment of the purpose of this review and as the nature of the matter commands the studies used were primary descriptive observational studies and secondary studies.

Despite being acquired twenty-six publications of case series or case reports that expose information on seventy-one children only thirtyfive of the cases met the inclusion criteria. Clinical cases sample is reduced not only by the usage of inclusion and exclusion criteria but also for searching only one primary database, being results generalization highly unlikely. For the results of upcoming studies might be generalized, such studies must focus on primary analytical observational evidence - using hospital medical records, for example and a larger number of electronic databases must be searched without language restriction.

The evidence of child maltreatment may be of other nature than medical. Whenever medical judgment was denied by court verdict the clinical case was considered as "suspect" - of "weak" evidence of maltreatment - and excluded. The lesions considered recognizable in dental office are intraoral lesions, signs or external lesions of the head or neck. Lesions elsewhere, even if bite marks, and fractures were rejected. This study stems from the assumption that in all cases described intraoral evaluation was done, having "intraoral" been defined as from the mucocutaneous junction of the lips till the hypopharynx. Injuries description in terms of colour, distribution and number was often inaccurate, with few paper including graphics or photographic records of the cases.

Despite the limitations of scientific evidence it was possible to analyse some characteristics of child maltreatment and lesions by maltreatment. The age of the children abused ranged between nine days (Stricker [39]) and thirteen years (Carrotte [27]), being the average age of 27, 19 months. Fourteen of these children were female 
(40\%) and twenty one were males (60\%). Lesion were distributed through the mouth, face, head and neck: $25.7 \%$ of the cases named intraoral lesions, $85.7 \%$ facial lesions, $17.1 \%$ head lesions and $8.6 \%$ neck lesions. Various types of lesions were described: traumatic absence of teeth, dental avulsions and intrusions, petechiae, ecchymosis, contusions, perforations, abrasion, hematomas, edema, scars, burns, subconjunctival haemorrhage, chemosis, hyphema, oral bleeding, nosebleeds and bloody otorrhea.

A description of four cases of Munchausen syndrome by proxy comprising signs and symptoms of head and neck justifies that this syndrome is taken into account in the assessment of possible child maltreatment during dental consultation.

\section{Conclusions}

Despite the limitations of scientific evidence we may conclude that intraoral region and craniofacial regions are home to multiple nonfatal lesions by child maltreatment, meeting other systematic reviews. The signs and lesions of the orofacial and crania regions associated with non-lethal child maltreatment are missing teeth due trauma, dental avulsions and intrusions, petechiae, ecchymosis, contusions, soft tissue perforations, abrasion, hematomas, edema, scars, burns, subconjunctival haemorrhage, chemosis, hyphema, oral bleeding, nosebleeds and bloody otorrhea. By non-fatal child maltreatment other intraoral lesions such as lip contusions, lacerations of the alveolar mucosa and gingiva, hard palate abrasion, frenulum lacerations, perforations of the pharynx, pharyngeal lacerations and excoriations, soft palate lacerations, lacerations and abrasion of the mouth floor might also be observed. As for the differential diagnosis between accidental and intentional trauma further studies are required.

\section{Acknowledgments}

Fundação Nacional para a Ciência e Tecnologia, Portugal - FCT under the project (PEst-OE/MAT/UI0006/2014).

\section{References}

1. Kellogg ND (2007) American Academy of Pediatrics Committee on Child Abuse and Neglect, Evaluation of suspected child physical abuse. Pediatrics 119: 1232-1241.

2. Crespo M, Andrade D, Alves AL, Magalhães T (2011) [The dentist's role in the child abuse: diagnosis and report]. Acta Med Port 24 Suppl 4: 939-948.

3. Somani R, Vinita K, Kumar D, Khaira J (2011) Child abuse and its detection in the Dental Office. J Indian Acad Forensic Med 33: 361-365.

4. Deputy S (2003) Shaking-impact syndrome of infancy. Semin Pediatr Neurol 10: 112-119.

5. Altimier L (2008) Shaken Baby Syndrome. J Perinat Neonat Nurs 22: 68-76.

6. Stirling J Jr, American Academy of Pediatrics Committee on Child Abuse and Neglect (2007) Beyond Munchausen syndrome by proxy: identification and treatment of child abuse in a medical setting. Pediatrics 119: 1026-1030.

7. Perepletchikova F, Kaufman J (2010) Emotional and behavioral sequelae of childhood maltreatment. Curr Opin Pediatr 22: 610-615.

8. Wong PY, How CH, Wong PC (2013) PILL series. Management of child abuse. Singapore Med J 54: 533-536.

9. Barber MA, Sibert JR (2000) Diagnosing physical child abuse: the way forward. Postgrad Med J 76: 743-749.
10. McDonald KC (2007) Child abuse: approach and management. Am Fam Physician 75: 221-228.

11. Lee AC (2008) Bruises, blood coagulation tests and the battered child syndrome. Singapore Med J 49: 445-449.

12. Woodman J, Lecky F, Hodes D, Pitt M, Taylor B, et al. (2010) Screening injured children for physical abuse or neglect in emergency departments: a systematic review. Child Care Health Dev 36: 153-164.

13. da Fonseca MA, Feigal RJ, ten Bensel RW (1992) Dental aspects of 1248 cases of child maltreatment on file at a major county hospital. Pediatr Dent 14: 152-157.

14. Jacobi G, Dettmeyer R, Banaschak S, Brosig B, Herrmann B (2010) Child abuse and neglect: diagnosis and management. Dtsch Arztebl Int 107: 231-239.

15. Ermertcan AT, Ertan P (2010) Skin manifestations of child abuse. Indian J Dermatol Venereol Leprol 76: 317-326.

16. Smith A (2011) Nonaccidental injury in childhood. Aust Fam Physician 40: 858-861.

17. Stephenson T (1997) Ageing of bruising in children. J R Soc Med 90: 312-314.

18. Pau-Charles I, Darwich-Soliva E, Grimalt R (2012) [Skin signs in child abuse]. Actas Dermosifiliogr 103: 94-99.

19. Carpenter SL, Abshire TC, Anderst JD; Section on Hematology/ Oncology and Committee on Child Abuse and Neglect of the American Academy of Pediatrics (2013) Evaluating for suspected child abuse: conditions that predispose to bleeding. Pediatrics 131: e1357-1373.

20. Maguire S, Mann MK, Sibert J, Kemp A (2005) Are there patterns of bruising in childhood which are diagnostic or suggestive of abuse? A systematic review. Arch Dis Child 90: 182-186.

21. Maguire S, Hunter B, Hunter L, Sibert JR, Mann M, et al. (2007) Diagnosing abuse: a systematic review of torn frenum and other intraoral injuries. Arch Dis Child 92: 1113-1117.

22. Sobel RS, Kerns DL (1983) A Case of child abuse. In: Sanger RG, Bross DC, eds. Clinical management of child abuse. Chicago: Quintessence 159-164.

23. Grace A, Grace S (1987) Child abuse within the ear, nose and throat. J Otolaryngol 16: 108-111.

24. Reinhart MA (1987) Sexual abuse of battered young children. Pediatr Emerg Care 3: 36-38.

25. Symons AL, Rowe PV, Romaniuk K (1987) Dental aspects of child abuse: review and case reports. Aust Dent J 32: 42-47.

26. Nazer H, Daradkeh T, Mohamed S, Shamayleh AQ, Marei O (1988) A diagnostic dilemma in Jordan: two child abuse case studies. Child Abuse Negl 12: 593-599.

27. Carrotte PV (1990) An unusual case of child abuse. Br Dent J 168: 444-445.

28. Hobbs CJ, Wynne JM (1990) The sexually abused battered child. Arch Dis Child 65: 423-427.

29. Manning SC, Casselbrant M, Lammers D (1990) Otolaryngologic manifestations of child abuse. Int J Pediatr Otorhinolaryngol 20: 7-16.

30. Willner A, Ledereich PS, de Vries EJ (1992) Auricular injury as a presentation of child abuse. Arch Otolaryngol Head Neck Surg 118: 634-637.

31. Barrett TG, Debelle GD (1995) Near-fatal aspiration of a child's dummy: design fault or deliberate injury? J Accid Emerg Med 12: 154-155.

32. Gustavson E, Levitt C (1996) Physical abuse with severe hypothermia. Arch Pediatr Adolesc Med 150: 111-112.

33. Mossakowska B (1996) Medical observations on child abuse. Child Abuse Negl 20: 141-143.

34. Kapoor S, Schiffman J, Tang R, Kiang E, Li H, et al. (1997) The significance of white-centered retinal hemorrhages in the shaken baby syndrome. Pediatr Emerg Care 13: 183-185.

35. Drack AV, Petronio J, Capone A (1999) Unilateral retinal hemorrhages in documented cases of child abuse. Am J Ophthalmol 128: 340-344. 
Citation: Banheiro R, Pereira EC, Pereira CP (2015) Injuries of Non-Lethal Child Physical Abuse to the Crania and Orofacial Regions: A Scientific Review. J Forensic Res 6: 285. doi:10.4172/2157-7145.1000285

Page 5 of 5

36. Ho WS, Ying SY, Wong TW (2000) Bizarre paediatric facial burns. Burns 26: 504-506.

37. Leonardi DF, Vedovato JW, Werlang PM, Torres OM (1999) Child burn: accident, neglect or abuse. A case report. Burns 25: 69-71.

38. Ly JQ (2002) Incidental finding of nonaccidental trauma in a patient reportedly found unconscious. J Emerg Med 23: 417-418.

39. Stricker T, Lips U, Sennhauser FH (2002) Oral bleeding: Child abuse alert. J Paediatr Child Health 38: 528-529.

40. Spitzer SG, Luorno J, Noël LP (2005) Isolated subconjunctival hemorrhages in nonaccidental trauma. J AAPOS 9: 53-56.

41. Krugman SD, Lantz PE, Sinal S, De Jong AR, Coffman K (2007) Forced suffocation of infants with baby wipes: a previously undescribed form of child abuse. Child Abuse Negl 31: 615-621.

42. Martin SA (2007) A case of tot maltreatment. J Pediatr Health Care 21 323-324, 350-3.
43. Yavuz MS, Buyukyavuz I, Savas C, Ozguner IF, Kupeli A, et al. (2008) A battered child case with duodenal perforation. J Forensic Leg Med 15: 259-262.

44. Pierce MC, Smith S, Kaczor K (2009) Bruising in infants: those with a bruise may be abused. Pediatr Emerg Care 25: 845-847.

45. Kondolot M, Yağmur F, Yıkılmaz A, Turan C, Öztop DB, et al. (2011) A life-threatening presentation of child physical abuse: jejunal perforation. Pediatr Emerg Care 27: 1075-1077.

46. Skarbek-Borowska SE, Campbell KT (2011) Globe rupture and nonaccidental trauma: two case reports. Pediatr Emerg Care 27: 544-546.

47. Gharib B, Esmaeili S, Mazloomi Nobandegani N, Shariati G (2012) Physical punishment, abuse, torture or revenge? A case report. Acta Med Iran 50: 580-582. 